Memoirs of the Faculty of Science, Kyushu University

Ser. A, Vol. 24, No. 2, 1970

\title{
A REMARK ON THE TRACE FORMULA FOR AN INSEPARABLE CORRESPONDENCE IN AN ALGEBRAIC FUNCTION FIELD
}

\author{
By \\ Tadashi WAshio \\ Dedicated to Professor Tosio Kitagawa on his 60-th birthday \\ (Received March 9, 1970)
}

1. Let $k$ be an algebraic function field of one variable with a perfect constant field $k_{0}$ of the characteristic $p>0$. H. Kappus [2] has constructed the non-trivial linear transformations of the vector space of differentials of the first kind in $k$ for inseparable correspondences and proved that the trace formula of their representations is equal to the fixed point formula.

If $k_{0}$ is especially a finite field $G F\left(p^{h}\right)$ and $k$ is an elliptic function field, we know well that it is possible to determine by the calculation of the number $N_{1}$ of the prime divisors of degree 1 in $k$ whether the Hasse invariant $A$ of $k$ is 0 or not. (M. Yamaguchi $[3] ;$ p. 33).

The purpose of this note is to prove that the relation between $A$ and $N_{1}$ is explicitly given by the formula

$$
A^{1+p+\cdots+p^{h-1}}=\overline{1-N_{1}},
$$

by means of the results of $H$. Kappus, where $\overline{1-N_{1}}$ denotes the residue class of $1-N_{1} \bmod p$ and it is identified with the element of $k_{0}$.

2. We shall quote here and in the next section the several wellknown results. We denote by $g$ the genus of $k$ and assume that $g>0$. Moreover, in this section, we assume that there exists a system of nonspecial prime divisors of $k$; i.e., there exists a system $\left\{\mathfrak{P}_{1}, \mathfrak{P}_{2}, \ldots, \mathfrak{P}_{g}\right\}$ of $g$ different prime divisors of degree 1 of $k$ such that the divisor class of $\mathfrak{P}_{1} \mathfrak{F}_{2} \ldots \mathfrak{P}_{g}$ is of dimension 1 .

Let $t_{i}$ be a prime element of $\mathfrak{P}_{i}$ for $1 \leqq i \leqq g$. The following proposition is due to Hasse ([1]; Satz 4). 
Proposition 1. There exist the elements $v_{1}, v_{2}, \ldots, v_{g}$ in $k$ satisfying the following conditions.

$$
v_{j} \equiv \frac{e_{i j}}{t_{i}^{p}}-\frac{a_{i j}}{t_{i}} \bmod \mathfrak{P}_{i}^{0} \text { for } 1 \leqq i, j \leqq g,
$$

where $e_{i j}=\left\{\begin{array}{ll}1 & \text { if } i=j \\ 0 & \text { if } i \neq j\end{array} \quad\right.$ and $a_{i j} \in k_{0}$.

$$
\operatorname{ord}_{\mathfrak{B}} v_{j} \geqq\left\{\begin{array}{ccc}
-p & \text { if } & \mathfrak{B}=\mathfrak{P}_{i} \\
0 & \text { if } & \mathfrak{P} \neq \mathfrak{P}_{i}
\end{array}\right.
$$

for a prime divisor $\mathfrak{P}$ of $k$, where $\operatorname{ord}_{\mathfrak{B}} v_{j}$ means the order of $v_{j}$ at $\mathfrak{P}$.

The $g$-rowed square matrix $A=\left(a_{i j}\right)$ generated by $a_{i j}$ in Proposition 1 is the so-called Hasse-Witt matrix associated with $\left\{\mathfrak{P}_{i}, t_{i}\right\}$.

Now we take a basis of the differentials of the first kind in $k$ and denote by $\left\{d u_{i}\right\},(1 \leqq i \leqq g)$, it. Then we can obtain the following representations for $d u_{i},(1 \leqq i \leqq g)$,

$$
\frac{d u_{i}}{d t_{j}} \equiv \sum_{\nu=0}^{p-1} b_{i j}^{(\nu)} t_{j}^{\nu} \bmod \mathfrak{P}_{j}^{p},
$$

where $b_{i j}^{(\nu)} \in k_{0}$ and it is uniquely determined by $d u_{i}, \Re_{j}$ and $t_{j}$ for $1 \leqq i$, $j \leqq g$.

We denote by $B_{0}$ and $B_{p-1} g$-rowed square matrices $\left(b_{i j}^{(0)}\right)$ and $\left(b_{i j}^{(p-1)}\right)$ respectively. Then we have the well-known fact. (Hasse [1]; Satz 6).

Proposition 2. The matrix $B_{0}$ is regular and the equality $A=B_{0}^{-1} B_{p-1}$ holds for the Hasse-Witt matrix $A$ of $k$.

3. We assume that $k_{0}$ is a finite field $G F\left(p^{h}\right)$. Let $k^{\prime}$ be a field which is isomorphic with $k$ over $k_{0}$ and algebraically disjoint with $k$ over $k_{0}$. Then we may assume that $k$ and $k^{\prime}$ are given by the forms $k=k_{0}(x, y)$ and $k^{\prime}=k_{0}\left(x^{\prime}, y^{\prime}\right)$ satisfying the same equation $f(x, y)=f\left(x^{\prime}, y^{\prime}\right)=0$, where the coefficients of $f$ belong to $k_{0}$.

The Frobenius correspondence $F$ of $k$ is associated with the prime ideal

$$
F_{k \times k^{\prime}}=k \times k^{\prime}\left(x-x^{\prime q}, y-y^{\prime q}\right)
$$


in the integral domain $k \times k^{\prime}$, where $q=p^{h}$.

If we denote by $K$ and $k^{\pi}$ the residue class fields modulo $F_{k \times k^{\prime}}$ of $k \times k^{\prime}$ and $k^{\prime}$ respectively, the equality $K=k^{\pi}=k^{q^{-1}}$ holds and so $K$ is purely inseparable and of finite degree $q$ over $k$. (Figure).

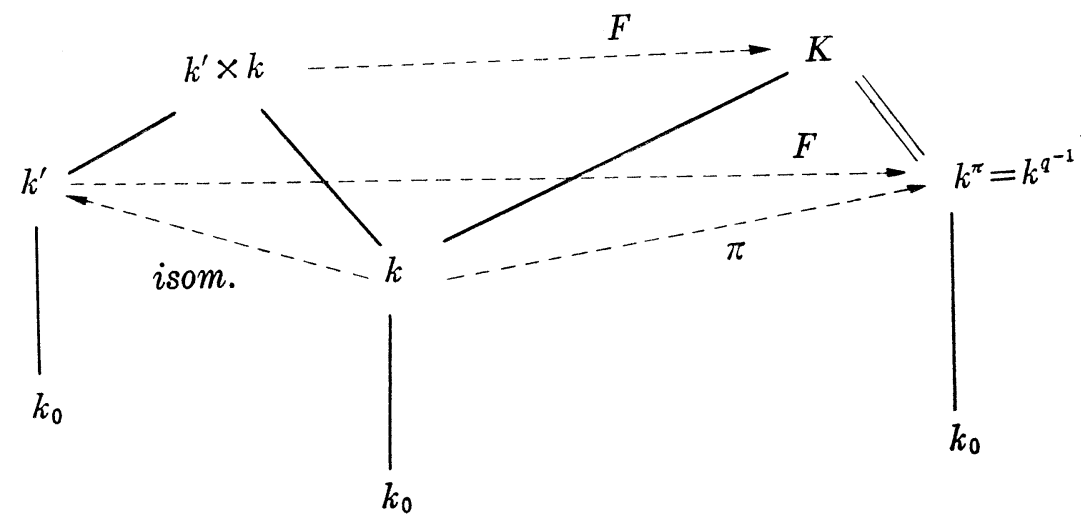

Fig.

$k^{q^{-1}}$ means the set of $q$-th roots of all elements of $k$.

Now, let $\omega$ be a differential of $k$. For the Frobenius correspondence $F$ of $k, H$. Kappus [2] has defined the $k_{0}$-linear transformation of the space of differentials of $k$ to itself by the rule

$$
\omega F=C^{h}\left(\omega^{\pi}\right)
$$

where $C$ denotes the Cartier-operator and $C^{h}$ means to act $C h$ times. $\omega^{\pi}$ means the image of $\omega$ for $\pi$ and it is a differential of $K=k^{\pi}=k^{q^{-1}}$.

If $\omega$ is of the first kind, $\omega F$ is so, since the mapping $\pi$ is the $k_{0}$-linear transformation of differentials of the first kind of $k$ into those of $k^{\pi}=K$ and the operator $C^{h}$ is the $k_{0}$-linear transformation of differentials of the first kind of $K$ into those of $k$.

Hence we can obtain a matrix representation $P$ of $F$ in the vector space of differentials of the first kind of $k$. The following proposition is due to H. Kappus ([2]; p. 382).

Proposition 3. Let $k$ be a field of algebraic functions of one variable; assume that the field of constants of $k$ is a finite field $G F\left(p^{h}\right)$. Denote by $N_{1}$ the number of prime divisors of degree 1 in $k$ and denote by $g$ the genus 
of $k$; assume that $g>0$.

Then, for a matrix representation $P$ of the Frobenius correspondence of $k$ in the vector space of differentials of the first kind of $k$, the equality

$$
\text { Trace } P=\overline{1-N_{1}}
$$

holds.

4. We assume that there exists a system of non-special prime divisors of $k$. We shall show that a representation of Frobenius correspondence $F$ of $k$ is explicitly obtained by the Hasse-Witt matrix. Let $\left\{d u_{i}\right\},(1 \leqq i \leqq g)$, be a basis of differentials of the first kind of $k$; fix it. Let $\left\{\mathfrak{P}_{j}\right\},(1 \leqq j \leqq g)$, be a system of non-special prime divisors of $k$ and let $t_{j}$ be a prime element of $\mathfrak{P}_{j},(1 \leqq j \leqq g)$; fix them.

We may assume that $k=k_{0}(x, y)$, where $x$ and $y$ are elements of $k$ which satisfy some irreducible equation $f(x, y)=0$ with the coefficients in $k_{0}$.

Then, for each integer $l,(1 \leqq l \leqq h)$, the field $k^{p^{-l}}$ coincides with $k_{0}\left(x^{p^{-l}}, y^{p^{-l}}\right)$, where $x^{p^{-l}}$ and $y^{p^{-l}}$ satisfy the irreducible equation $f^{p^{-l}}\left(x^{p^{-l}}\right.$, $\left.y^{p^{-l}}\right)=0 . \quad f^{p^{-l}}$ means the polynomial whose coefficients are the $p^{l}$-th roots of the coefficients of the polynomial $f$.

For every element $z$ of $k$, the correspondence $z \rightarrow z^{p^{-l}}$ gives an isomorphism of $k$ onto $k^{p^{\prime \prime}}$. This isomorphism gives naturally the transformations of divisors and differentials of $k$ into those of $k^{p^{-l}}$.

It follows that the image $\left\{\mathfrak{P}_{j}^{(l)}\right\}$ of $\left\{\mathfrak{P}_{j}\right\}$ is a system of non-special prime divisors of $k^{p^{-l}}$ and the image $\left\{d u_{i}^{(h-l)}\right\}$ of $\left\{d u_{i}\right\}$ is a basis of differentials of the first kind of $k^{p^{-l}}$. Obviously the image $t_{j}^{(l)}$ of $t_{j}$ coincides with $t_{j}^{p-l}$ and it is a prime element of $\mathfrak{P}_{j}^{(l)}$.

Moreover, we see easily that the image $A^{p^{-l}}=\left(a_{i j}^{p^{-l}}\right)$ of the Hasse-Witt matrix $A=\left(a_{i j}\right)$ associated with $\left\{\mathfrak{P}_{j}, t_{j}\right\}$ in $k$ is also the Hasse-Witt matrix associated with $\left\{\mathfrak{P}_{j}^{(l)}, t_{j}^{p^{-l}}\right\}$ in $k^{p^{-l}}$. If we denote by $d u_{i}^{\pi}, \mathfrak{P}_{j}^{\pi}$ and $t_{j}^{\pi}$ the images of $d u_{i}, \mathfrak{P}_{j}$ and $t_{j}$ for $F$ respectively, the equalities $d u_{i}=d u_{i}^{(0)}$, $\mathfrak{P}_{j}^{\pi}=\mathfrak{P}_{j}^{(h)}$ and $t_{j}^{\pi}=t_{j}^{p^{-h}}$ hold.

Now we shall find the matrix representation $P$ of $F$ with respect to the basis $\left\{d u_{i}\right\}$. We may assume that the differential $d u_{i}$ has the representation

$$
d u_{i} \equiv \sum_{\nu=0}^{p-1} b_{i j}^{(\nu)} t_{j}^{\nu} d t_{j} \bmod \mathfrak{P}_{j}^{p}
$$


where $b_{i j}^{(\nu)} \in k_{0}$ for $1 \leqq i, j \leqq g, 0 \leqq \nu \leqq p-1$.

Then, on the one hand, the congruence

$$
d u_{i}^{(h-l)} \equiv \sum_{\nu=0}^{p-1}\left(b_{i j}^{(\nu)}\right)^{p^{-l}} t_{j}^{p^{-l} \nu} d t_{j}^{p^{-l}} \bmod \quad \mathfrak{P}_{j}^{\left(l^{p}\right.}
$$

holds for $1 \leqq l \leqq h, 1 \leqq i, j \leqq g$.

It follows obviously that for the Cartier-operator $C$ the congruence

$$
C\left(d u_{i}^{(h-l)} \equiv\left(b_{i j}^{(p-1)}\right)^{p^{-l}} d t_{j}^{p^{-(l-1)}} \bmod \mathfrak{P}_{j}^{(l-1)}\right.
$$

holds for $1 \leqq i, j \leqq g$.

On the other hand, there exist the elements $s_{i n}^{(h-(l-1))},(1 \leqq i, j \leqq g)$, in $k_{0}$ such that

$$
C\left(d u_{i}^{(h-l)}\right)=\sum_{n=1}^{g} s_{i n}^{(h-(l-1))} d u_{n}^{(h-(l-1))}
$$

since $C\left(d u_{i}^{(h-l)}\right)$ is a differential of the first kind of $k^{p^{-(l-1)}}$ and $d u_{n}^{(h-\langle l-1)\rangle}$, $(1 \leqq n \leqq g)$, is a basis of such differentials.

So the other congruence

$$
C\left(d u_{i}^{(h-l)}\right) \equiv \sum_{n=1}^{g} s_{i n}^{(h-(l-1))}\left(b_{n j}^{(0)}\right)^{p^{-(l-1)}} d t_{j}^{p-(l-1)} \bmod \mathfrak{F}_{j}^{(l-1)}
$$

holds for $1 \leqq i, j \leqq g$. Thus we obtain

$$
\left(b_{i j}^{(p-1)}\right)^{p^{-l}}=\sum_{n=1}^{g} s_{i n}^{(h-(l-1))}\left(b_{n j}^{(0)}\right)^{p^{-(l-1)}} ; \text { i. e. }
$$

if we denote by $S_{h-(l-1)}, B_{0}$ and $B_{p-1}$ the matrices $\left(s_{i j}^{(h-(l-1))}\right),\left(b_{i j}^{(0)}\right)$ and $\left(b_{i j}^{(p-1)}\right)$ respectively, the equality

$$
B_{p-1}^{p l}=S_{h-(l-1)} B_{0}^{p-(l-1)}
$$

holds, where $B^{p^{-l}}$ means the matrix $\left(b_{i j}^{p^{-l}}\right)$.

It is rewritten by

$$
S_{h-(l-1)}=B_{p-1}^{p^{-l}}\left(B_{0}^{-1}\right)^{p^{-(l-1)}},
$$

since $B_{0}$ is a regular matrix from Proposition 2. So we obtain inductively 


$$
\begin{aligned}
& P\left[\begin{array}{c}
d u_{1} \\
\vdots \\
d u_{g}
\end{array}\right]=\left[\begin{array}{c}
C^{h}\left(d u_{1}\right) \\
\vdots \\
C^{h}\left(d u_{g}\right)
\end{array}\right]=\left\{B_{p-1}^{p^{-h}}\left(B_{0}^{-1}\right)^{p^{-(h-1)}}\right\} \cdots \\
& \cdots\left\{B_{p-1}^{p^{-l}}\left(B_{0}^{-1}\right)^{\left.p^{-(l-1}\right)}\right\} \cdots\left\{B_{p-1}^{p^{-1}} B_{0}^{-1}\right\}\left[\begin{array}{c}
d u_{1} \\
\vdots \\
d u_{g}
\end{array}\right] .
\end{aligned}
$$

Thus we have

$$
\begin{aligned}
& P=\left\{B_{0}^{p^{-h}} A^{p^{-h}}\left(B_{0}^{-1}\right)^{p^{-(h-1)}}\right\} \cdots \\
& \cdots\left\{B_{0}^{p^{-l}} A^{p^{-l}}\left(B_{0}^{-1}\right)^{p^{-(l-1)}}\right\} \cdots\left\{B_{0}^{p^{-1}} A^{p^{-1}} B_{0}^{-1}\right\} \\
& =B_{0}^{p^{-h}} A^{p^{-h}} \cdots A^{p^{-l}} \cdots A^{p^{-1}} B_{0}^{-1} \\
& =B_{0} A A^{p} \cdots A^{p^{h-1}} B_{0}^{-1},
\end{aligned}
$$

since $B_{p-1}=B_{0} A$ holds from Proposition 2 .

Therefore we obtain the equality

$$
\text { Trace } P=\operatorname{Trace}\left(A A^{p} \ldots A^{p^{h-1}}\right) \text {. }
$$

This means that Trace $\left(A A^{p} \ldots A^{p^{h-1}}\right)$ is independent on the choice of $\left\{\mathfrak{P}_{j}, t_{j}\right\}$.

Hence, by making use of Proposition 3, we have following results.

Theorem. Let $k$ be a field of algebraic functions of one variable; assume that the field of constants of $k$ is a finite field $G F\left(p^{h}\right)$. Denote by $g$ the genus of $k$; assume that $g>0$. Moreover assume that there exists a system of non-special prime divisors of $k$.

Then the equality

$$
\operatorname{Trace}\left(A A^{p} \cdots A^{p^{h-1}}\right)=\overline{1-N_{1}}
$$

holds for the number $N_{1}$ of prime divisors of degree 1 and the Hasse-Witt matrix $A$.

Corollary. Let notations and assumptions be as in the above Theorem. If $k$ is an elliptic function field, the equality

$$
A^{1+p+\cdots+p^{h-1}}=\overline{1-N_{1}}
$$

holds; i.e., a necessary and sufficient condition for $A=0$ is $N_{1} \equiv 1 \bmod p$. 
A Remark on the Trace Formula for an Inseparable Correspondence in an Algebraic Function Field

\section{Bibliography}

[1] H. HASSE und E. WITT, Zyklische unverzweigte Erweiterungskörper vom Primzahlgrade $p$ über einem algebraischen Funktionenkörper der Charakteristik $p$, Monatshefte für Mathematik und Physik, 43 (1936), 477-492.

[2] H. KAppus, Eine Spurformel für inseparable Korrespondenzen algebraischer Funktionenkörper, Archiv der Mathematik, 18 (1967), 378-382.

[3] M. Yamaguchi, Daenkyokusen no jundōkeikan ni tuite, Sūgaku (in Japanese), 14 (1962) , 30-33. 